\title{
EUV detection system with calibrated responsivity
}

\author{
J. Mikolajczyk \& Z. Bielecki \\ Institute of Optoelectronics, Military University of Technology, Poland
}

\begin{abstract}
We describe a measuring system of EUV radiation energy using special calibration responsivity. This system is specified by responsivity of $0.043 \mathrm{~A} / \mathrm{W}$ at the wavelength range of $13.5 \pm 0.5 \mathrm{~nm}(\mathrm{FWHM}=7.2 \%)$. A determination of the calibration responsivity for a laser-plasma source with $\mathrm{Xe} / \mathrm{He}$ gas puff target is also discussed. This parameter makes it possible to reference measurement results to the standard wavelengths band of $13.5 \pm 0.13 \mathrm{~nm}(\mathrm{FWHM}=2 \%)$. The value of the calibration responsivity depends on the spectra of measured radiation and features of the system elements. In this paper, the analysis and investigation results of the source and the measuring system are demonstrated. Keywords: EUV radiation, optical sensors, energy meter.
\end{abstract}

\section{Introduction}

Accurate measurements of energy radiation are very important in nanolithography. The resists or masks can be damaged due to the measurements errors. Nowadays, energy meters of EUV radiation are also used for testing of lithography tools. The investigations are connected with characterization of EUV radiation sources, features of optical elements, etc. The responsivity and measurement accuracy are the main features of the measuring devices. The parameters are referenced to a standard wavelength range of $13.5 \pm 0.13 \mathrm{~nm}$. The range corresponds with the $\mathrm{FWHM}=2 \%$.

The developed commercial meters are usually built from multilayer mirrors and semiconductor detectors [1]. The mirrors simplify construction of the instruments. Using photodiodes makes it possible to achieve a high efficiency and plain measurements procedures. The pulse energy of radiation is determined by the measured charge generated in a photodiode, the value of the detector 
responsivity and the features of optical elements. Achievement of appropriate selectivity of measuring spectrum $(\mathrm{FWHM}=2 \%)$ is important issue of construction process of the meters.

The special mirrors with wavelength band of $2 \%$ have been constructed. Analyses and investigations showed that the mirrors are characterized by low reflectivity [2]. As the amount of photons reaching detector is limited, the instrument responsivity is decreased.

The described effect is avoided using the optical elements with broader wavelength range and a special calibration factor. This factor provides to refer measured data taken in the broader spectrum to the arbitrary one. The factor value depends on spectra of the measured radiation and some performances of the instrument elements. Basing on this factor and the meter responsivity, the calibrated responsivity is calculated by

$$
\mathrm{S}_{\mathrm{S}}=\mathrm{C}_{\lambda} \mathrm{S}_{\lambda} \text {. }
$$

The construction of the laser-plasma source with gas-puff target necessitated application of an appropriate system for energy measurement [3]. The features of the commercial meters (their dimensions and prices) forced to design a special measuring system (M-EUV system) [4]. The small size, the high-calibrated responsivity and the fully automated measuring process, all are the main system advantages. M-EUV system uses Mo/Si multilayer mirror (FWHM=7.3\%), silicon photodiode with integrated absorption filter and the calibration factor.

To the best of analysed knowledge, this work presents the experimental results of the first investigation of the system with calibrated responsivity used for measurements of energy emitted by the laser plasma source with $\mathrm{Xe} / \mathrm{He}$ gas puff target.

\section{Calibrated responsivity}

The determined value and the measured range of wavelengths describe the energy of source radiation using M-EUV system. The spectrum influence on measurement results is taken into consideration by determination of the system responsivity. The responsivity is the most important parameter of absolute energy measurements and it is defined by

$$
\mathrm{R}_{\mathrm{D}}=\frac{\Delta \mathrm{I}_{\mathrm{ph}}}{\mathrm{P}_{\lambda}},
$$

where $I_{p h}$ is the current signal generated in detector and $P_{\lambda}$ is the power of the exposing radiation.

The spectral characteristic of M-EUV system responsivity depends on the mirror reflectivity and the detector responsivity. This characteristic is given by

$$
\mathrm{S}_{\lambda}=\mathrm{S}_{\text {Det }} \mathrm{R}_{\text {Mir }} \text {. }
$$

Theoretically determined characteristic of the system responsivity is shown in Fig. 1 . 


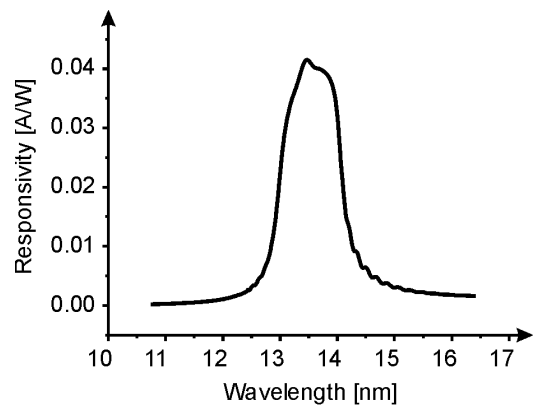

Figure 1: Theoretical characteristic of the system responsivity [5].

The maximum value is $0.043 \mathrm{~A} / \mathrm{W}$ at the wavelength range of $13.5 \pm 0.5 \mathrm{~nm}$. Analyses and investigations of the spectra generated from $\mathrm{Xe} / \mathrm{He}$ gas-puff target gave an opportunity to calculate the calibrated responsivity of the M-EUV system. In Fig. 2, it is presented the spectrum of the source radiation in the two ranges of wavelengths $13.5 \pm 0.5 \mathrm{~nm}$ and $13.5 \pm 0.13 \mathrm{~nm}$ describing the system responsivity.

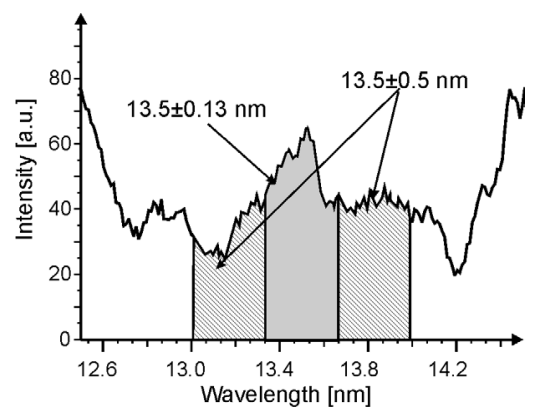

Figure 2: Radiation spectrum with analysed ranges of wavelength [5].

The marked areas specify the radiation energies in the chosen wavelength ranges. The energies are connected with the charge generated in the irradiated photodiode. The value of this charge is given by

$$
Q=\Omega \int_{0}^{\infty} E(\lambda) S_{\lambda} d \lambda,
$$

where $\Omega$ is the solid angle of the source radiation.

The energy value of radiation at the range of wavelengths of $13.5 \pm 0.13 \mathrm{~nm}$ is determined by

$$
E_{2 \%}(\lambda)=\frac{Q}{\Omega \int_{0}^{\infty} \mathrm{I}(\lambda) \mathrm{S}_{\lambda} \mathrm{d} \lambda}
$$


where $I(\lambda)$ is the normalized function characterising the spectrum of radiation and it is described by

$$
\int_{13.5 \pm 0.13 \mathrm{~nm}} \mathrm{l}(\lambda) \mathrm{d} \lambda=1
$$

The expression given by

$$
S_{S(\lambda)}=\int_{0}^{\infty} I(\lambda) S_{\lambda} d \lambda,
$$

defines the calibration responsivity of the M-EUV system [1]. This responsivity depends on features of system elements and the source spectra. The spectra can be shifted by changing conditions of the plasma generation in the investigated source. The aforesaid conditions concern a delay of the nozzles valve opening with respect to a laser pulse, power density of $\mathrm{Nd}$ :YAG laser radiation on the target surface, position of a laser beam focus with respect to the gas target axis, pressure of the remaining gases in the source chamber, gases pressures in the valve nozzles [6].

\section{Measurement method}

Measurements of the calibration responsivity were taken using the laser plasma source with $\mathrm{Xe} / \mathrm{He}$ gas puff target and the model instrument (E-Mon energy meter [1]). The main aim of the investigations was determination of energy radiation in two ranges of wavelengths $13.5 \pm 0.27 \mathrm{~nm}$ and $13.5 \pm 0.5 \mathrm{~nm}$. The ranges are defined by spectral characteristics of mirrors reflectivity used in E-Mon meter and M-EUV system.

The calibration responsivity $\left(S_{S E-M o n}\right)$ and measurement uncertainty of E-Mon meter referenced to the wavelength range of $13.5 \pm 0.13 \mathrm{~nm}$ is taken into consideration for determination of the M-EUV system responsivity.

The calibration factor $\left(C_{M-E U V}\right)$ is determined by measured energies with EMon meter and M-EUV system. The value of the factor is given by

$$
C_{M-E U V}=\frac{E_{7 \%}}{E_{3 \%}},
$$

where $E_{7 \%}$ and $E_{3 \%}$ are the energy values in the wavelength ranges of $13.5 \pm 0.5 \mathrm{~nm}$ and $13.5 \pm 0.27 \mathrm{~nm}$.

This factor makes it possible to specify the calibrated responsivity of M-EUV system referenced to $\mathrm{FWHM}=2 \%$. This responsivity is calculated by

$$
\mathrm{S}_{\mathrm{S} M-\mathrm{EUV}}=\mathrm{C}_{\mathrm{M}-\mathrm{EUV}} \mathrm{S}_{\mathrm{S} \text { E-Mon }} \text {. }
$$

\section{Experimental results}

The measurements of the source energy and determination of the energies ratio in two wavelength ranges were the main aim of the investigations. The researches defined the calibration responsivity and also the calibration factor of 
M-EUV system. The spectra of the radiation emitted by the laser-plasma source with $\mathrm{Xe} / \mathrm{He}$ gas puff target were also analysed. The measurements were taken for different conditions of plasma generation in the source.

\subsection{Influence of delay of Xe/He nozzles valve opening}

The delay of the nozzles valve opening with respect to a laser pulse has direct impact on a dimension and density of the gas target. Measured characteristics of the energy and the calibration factor as a function of the delay of Xe and Xe gas nozzles are shown in Fig. 3.
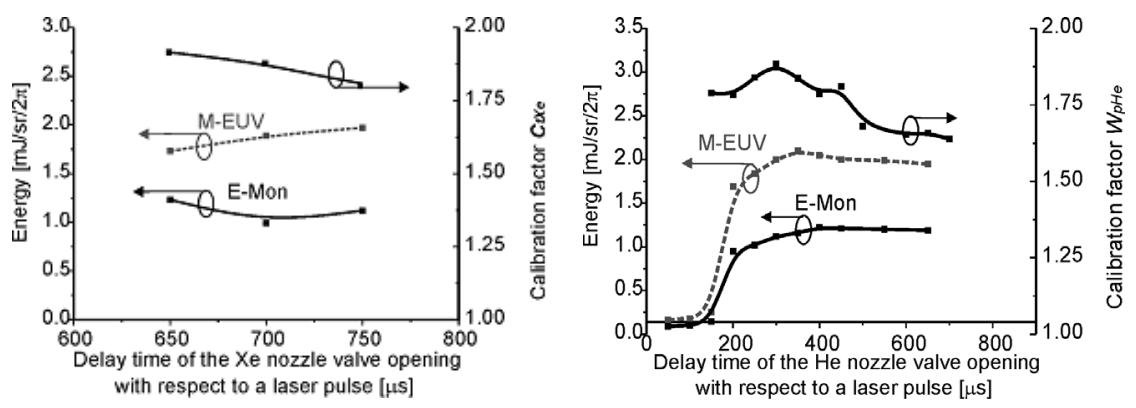

Figure 3: Energy radiation and the calibration factor at various delays of Xe and Xe gas nozzles.

The value changes of the calibration factor with delay of Xe gas nozzle are up to relative value of $10 \%$. Its mean value is $1.85 \pm 0.06$. The maximum energy emitted by the source was achieved for delay from 650 to $850 \mu \mathrm{s}$.

For He gas, a threshold delay was observed. The energy drops off dramatically for the delay of $200 \mu$ s. The noticed threshold has no influence on the factor characteristic.

\subsection{Influence of $\mathrm{Xe} / \mathrm{He}$ gases pressures in the valve nozzles}

The gases pressure similar to nozzles delay forms the target performances. This influence concerns the source spectra. The characteristic changes of energy and calibration factor as a function of the pressures are shown in Fig. 4.

The increase in the energy radiation is determined by simultaneous increase in the Xe gas pressure. The maximum value of energy is limited by strength of gas pipes. The calibration factor characteristic follows the energy characteristics.

For $\mathrm{He}$ gas pressure below $0.5 \mathrm{MPa}$, the optimal range of energies is observed. In this case, the calibrated factor decreases negligibly with He gas pressure.

\subsection{Influence of pressure of the remaining gases}

The EUV radiation is absorbed not only by a solid body but also by a gaseous one. The remaining gas target can be accumulated in the chamber of the laserplasma source. The pressure of the remaining gas depends on the source 
repetition and the efficiency of the vacuum pump. The measured value of the pressure was shifted from $10^{-6}$ to $10^{-2}$ mbar at the source repetition of $10 \mathrm{~Hz}$. The changes of energy and the calibration factor for the remaining gases are shown in Fig. 6.
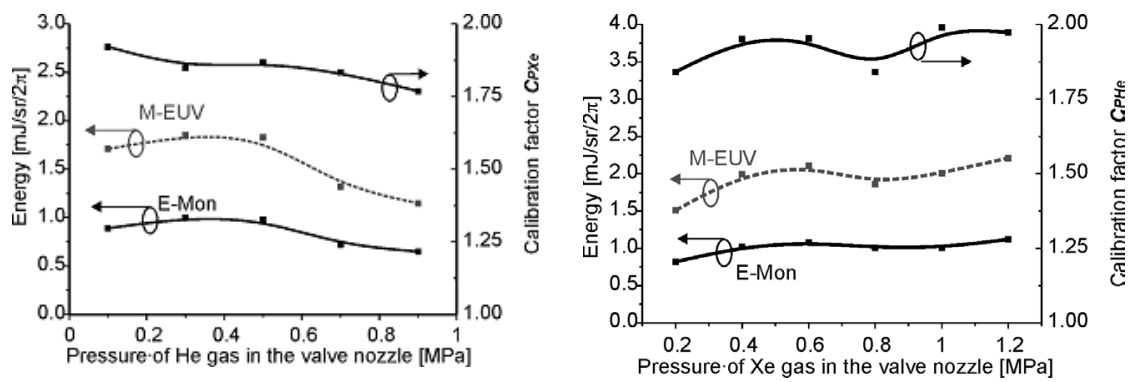

Figure 4: Energy radiation and the calibration factor vs. $\mathrm{Xe} / \mathrm{He}$ gases pressures in the valve nozzles.
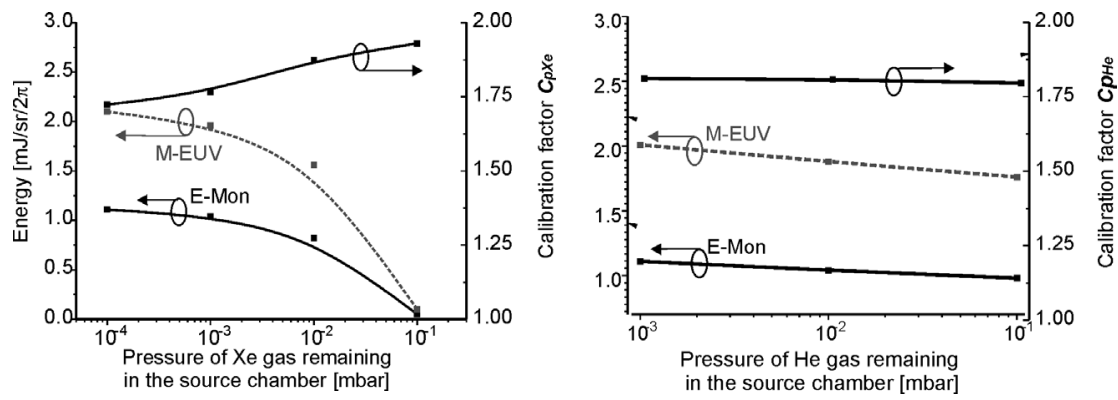

Figure 5: Energy and the calibration factor as a function of pressure of the remaining gases in the source chamber.

The energy and the calibration factor vary significantly with the Xe remaining gas. The threshold pressure of the energy dropping is observed near the value of $10^{-2}$ mbar. The calibration factor increases with the pressure build-up. Its mean value is $1.82 \pm 0.06$. For the $\mathrm{He}$ gas, the described influences are not so noticeable. The mean value of the calibrated factor is $1.83 \pm 0.03$.

\subsection{Influence of position of a laser beam focus}

The valve construction makes it possible to control a beam focus position on the gas target space. Figure 6 shows energy and calibration factor characteristics varied with a position of a laser beam focus.

The measured energy and the calibration factor decrease with shifting the target position more than value of $+10 \mu \mathrm{m}$. The mean value of the calibrated factor was $1.77 \pm 0.06$. 


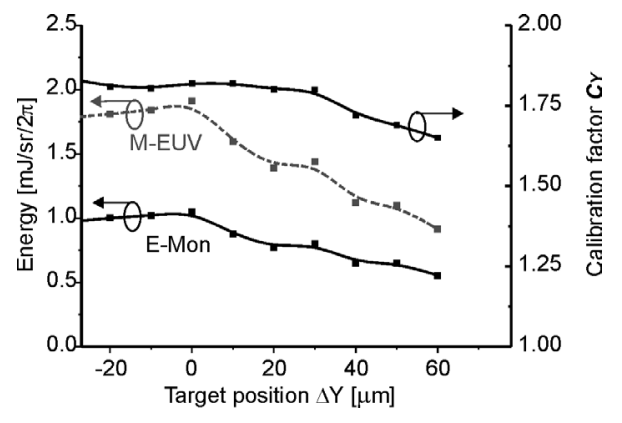

Figure 6: Energy and the calibration factor as a function of positions of a laser beam focus.

\subsection{Influence of energy radiation of Nd:YAG laser}

The Nd:YAG laser with output energy adjustment was used during the investigation procedures. The energy control was executed by changing a duration time of the active laser pumping. This time can be adjusted in the range from 200 to $2000 \mu \mathrm{s}$. Figure 7 shows the source energies and the calibrated factor for changes of duration time of the active laser pumping.

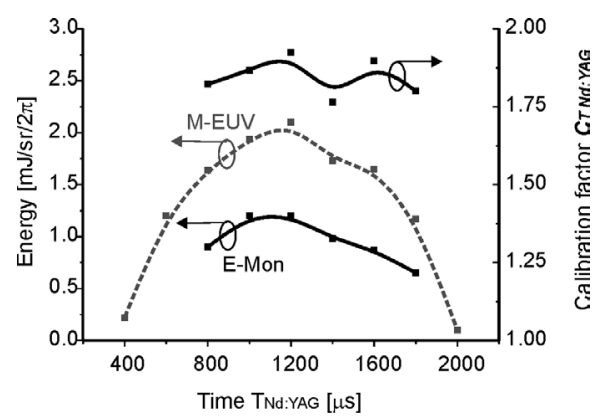

Figure 7: Energy and the calibration factor vs. duration time of the laser pumping.

\section{Results verification}

The results were verified by spectral measuring of the source radiation. The analysed spectra were taken by the spectrometer consisted of the reflecting grating (HITACHI 1200 1/mm) and CCD camera (Roper Scientists).

The values of the calibrated factor were calculated using the processed graphs of the measured spectra. The graphs were given by multiplication of the spectral characteristics of multilayer mirrors (used in the E-Mon meter and M-EUV system) and the source spectra. The example of the analysed graph is shown in Fig. 8 . 


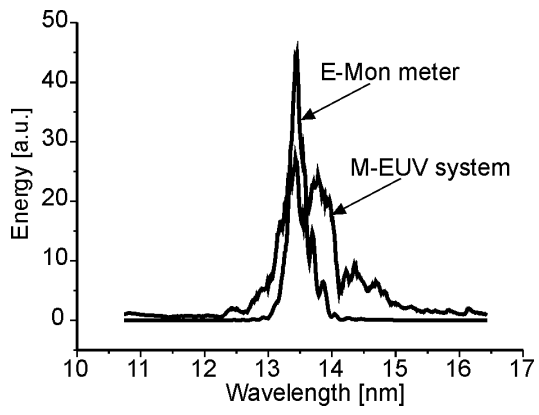

Figure 8: Resultant graphs of the spectra detected by the E-Mon meter and M-EUV system.

The maximum differences between the data taken by the measuring instruments and the spectral analyses were observed for changes of position of a laser beam focus $(12 \%)$, pressure of the remaining gases $(9.5 \%)$, and delay of the Xe nozzle valve opening $(8.8 \%)$. For other conditions of the plasma generations, the noticed differences were less than $4 \%$.

\section{Conclusions}

The paper presents a unique system for energy measurement of radiation emitted by the laser-plasma source with Xe/He gas puff target. The experimental results specified not only the features of the system, but also characterized an influence of the conditions of the plasma generation on the source spectra. The measured responsivity of the system is $0.043 \mathrm{~A} / \mathrm{W}$ in the range of wavelengths of $13.5 \pm 0.5 \mathrm{~nm}$.

For the maximum efficiency of the source, the calculated value of the calibrated factor is $1.79 \pm 0.03$ in the wavelength range of $13.5 \pm 0.13 \mathrm{~nm}$ hence the system responsivity of $0.291 \pm 0.027 \mathrm{~A} / \mathrm{W}$. Using the mirrors with wider spectral characteristic of the reflectivity (worse selectivity) has improved the measuring performance of the system.

Acceptance of the constant value of the calibration factor (independent on conditions of the plasma generation) is able to make the measurement error of $14 \%$. The uncertainty of factor calculation was $7.4 \%$. The relative value of the determined uncertainty of energy measurement in the spectrum of FWHM $=2 \%$ is $10.1 \%$. The presented system provides a simple, reliable solution to EUV energy measurements. That is why it is promising alternative for commercial meters.

\section{Acknowledgements}

This work was financially supported by the Polish Ministry of Science and Higher Education in the frame of research project No 0004/T00/2005/29. The authors would like to thank prof. H. Fiedorowicz (Institute of Optoelectronics, MUT, Warsaw) for fruitful discussion and advice. 


\section{References}

[1] Cross-Calibration of Extreme Ultraviolet (EUV) Energy Sensors International SEMATECH Technology Transfer \#04024498A-TR http://www.sematech.org/docubase/document/4498atr.pdf.

[2] http://www.iof.fhg.de/departments/optical-coatings/vuv-euv-ray/projects /euv_schmalband_e.html.

[3] Kranzusch S. \& Mann K. Spectral characterization of EUV radiation emitted from a laser-irradiated gas puff target, Optics Communications, vol. 200, Issue: 1-6, pp. 223-230, December 15, 2001.

[4] Mikolajczyk J., Bielecki Z. \& Wojtas J. Testing system for extreme ultraviolet detectors, Processing of SPIE, vol. 5948, pp. 594822-(1-9), April, 2005.

[5] Bielecki Z. \& Mikolajczyk J. Energy meter for Xe/He gas-puff laser plasma source, Optica Applicata, 2007 in print.

[6] Fiedorowicz H. \& et al. Compact laser plasma EUV source based on a gas puff target for metrology applications, Journal of Alloys and Compounds, vol. 401, Issue: 1-2, pp. 99-103, September 29, 2005. 